\title{
Physical Database Port to Workstations \\ Project Plan \\ Version 2.6
}

Date of Last Revision July 29, 1991

Clifford E. Rhoades, Jr.

Principal Author

University of California

Lawrence Livermore National Laboratory

Livermore, CA 94550

March 1993

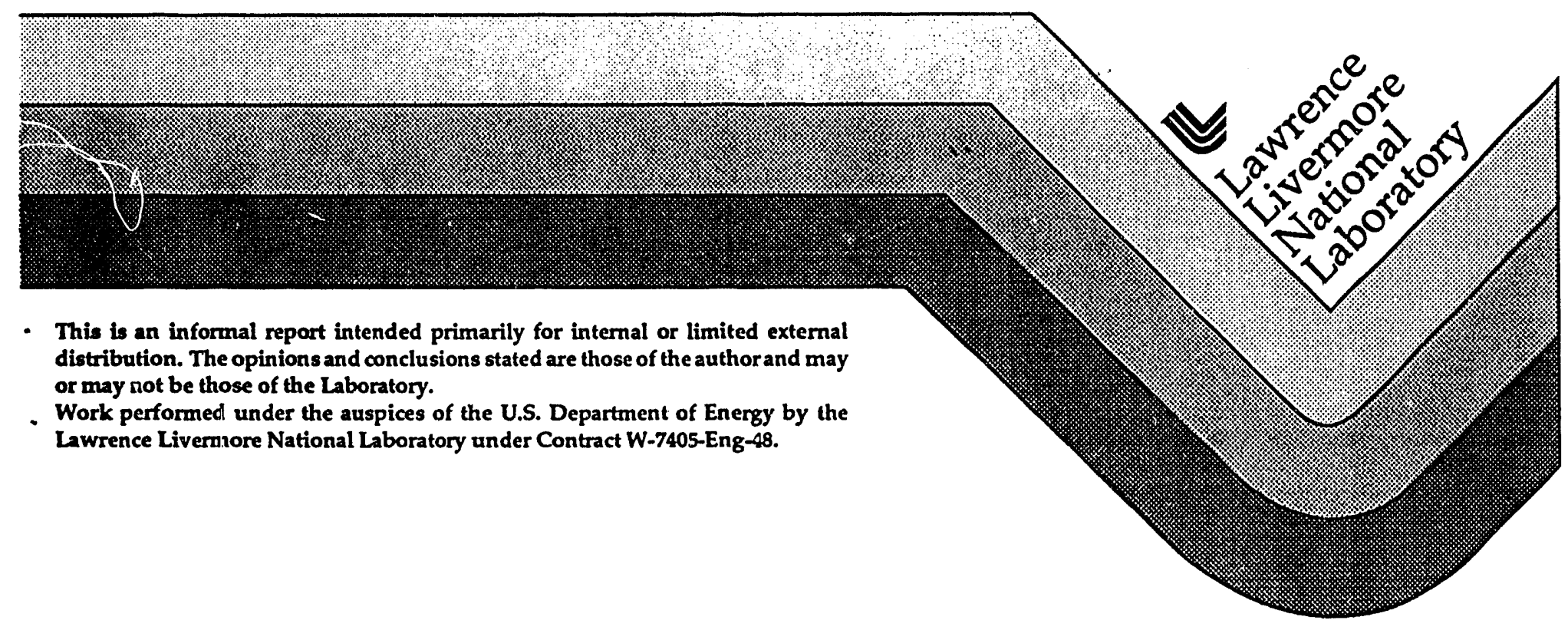




\section{DISCLAIMER}

This document was prepared as an acocount of work eponsored by an agency of the United States Government. Neither the United States Covernment nor the Univeraty of Callfornia nor any of their employeen, makes any warranty, express or implled; or assumes any legal liability or responsibility for the accuracy, completeness, or usefuiness of any information, apperratus, product, or process disclosed, or represents that its use would not infiringe psivately own rights. Reference hereln to any epecific commencial products, process, $\alpha$ cervice by trade name, trademark, manufectures, or otherwise, does not necesearily constitute or imply it endorsement, recommendation, or favoring by the United States Covernment or the University of California. The viewe and opinions of authors expresed herein do not necescarily state or reflect those of the United States Covernment or the Univeralty of Callfornia, and shall not be used for adventising or product endorsement purposea.

This report has been reproduced directly from the best available oopy. .

Available to DOE and DOE contractors from the Office of Scientific and Technical Information

P.O. Box 62, Oak Ridge IN 37831

Prices available from (615) 576-8401, FTS 626-8401

Available to the public from the

National Technical Information Service

US. Department of Commerce

5285 Port Royal Rd.

Springfield, VA 22161 


\section{Physical Database Port to Workstations \\ Project Plan \\ Version 2.6}

\section{Date of Last Revision}

July 29, 1991

\section{Clifford E. Rhoades, Jr. Principal Author}

\section{Table of Contents}

1. Project Goal

2. Project Objectives

2.1 Maintain Current Capabilities

2.2 Computational Accuracy

2.3 Computational Speed

2.4 Cray and Workstation Portability

2.5 Archival Requirements

2.6 Code Developer and Data Manager Education

2.7 Programming Standards

3. Implementation Order

4. Alternative Approaches

5. Criteria for Evaluating Approaches

6. Plan of Attack

6.1 Executive Overview

6.2 Port Intrinsics

6.3 Milestones

Appendix: Currently Known Constraints

Appendix: Miscellaneous Details which impact time estimates

Appendix: Eos4 Details

Appendix: Txs Details

Appendix: External Reference Specification of Supporting Library

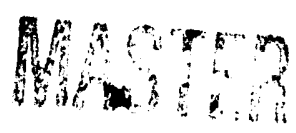




\section{Project Goal}

The project goal is to port those physical databases used on the Cray by our important production codes to high-performance Unix wurkstations while maintaining the current computational capabilities and accuracies, and achieving reasonably efficient execution on the workstations. The port must strike a judicious balance between (a) not changing the current N/LTSS databases, accessing libraries, generating codes and using codes, and (b) adversely impacting the maintenance or performance of the various codes that create or use the databases on the Cray. (Because of its forthcoming delivery, the Sun Sparcstation 2, using SunOS 4.0.3 or later, is the initial hardware platform selected for the first workstation port.) Our purpose in undertaking this project is to enable the production codes, Tart, Lasnex, Meg, Xraser, Sandyl (and its planned successor), Nike3d and Dyna3d to get up and running on the Unix platforms as soon as possible.

\section{Project Objectives}

To quantify project progress and completion, and to insure adequate communications among those involved in the project, directly or indirectly, a series of objectives have been developed. Objectives state generally what is to be provided and not the implementation methods. The latter are addressed later in this document.

Specifically excluded from this project are (a) conversion of the physical database generator codes (these are the responsibility of their owner-developers), (b) conversion of tools for users to inquire about databases on their Unix platform (these are the responsibility of their owner-developers) and (c) any new capabilities or further developments unneeded by the porting project (these are again the responsibility of the usual support mechanisms). Thus, it must be understood that this project does not address the general physical database problem and does not solve "next year's problems." Hence, there may well be additional burden on the current database developers who will retain responsibility for maintenance and update but may find that the tools on the Unix mainframes will be limited and that the conventions and programming restrictions may not be what they desire. These individuals and their supervisors should review this document carefully. It appears that certain physical database support mechanisms may be currently inadequate and that in some cases the support mechanisms are nonexistent or unidentified!

\subsection{Maintain Current Capabilities}

To allow continuation of the present operational mode and to insure a smooth transition from the Cray to the workstation environment, all required databases and associated access libraries will have their current capabilities in the new environment. Library routine specifications will be identical on the Cray and workstations. The databases will, at minimum, have the same physical content. The execution of database dependent production codes will be identical on the Cray and workstations, at least in so far as the data are concerned. Note that in some cases, it may be necessary to change the library accessing routines external specification. In which case, the Cray version will also be changed so that the library routine specifications will be identical on the Cray and workstations.

Since most Cray file formats are not available on the workstations, the workstation databases and their libraries may have to use a variety of techniques to provide the same capabilities. The project's primary approach will be to support either an ascii portable format (where this is readily feasible) or a bit-for-bit Cray identical absolute binary format (where ascii is not available or suitable). The physical databases are identified in the following Table 1. 
TABLE 1. The various physical databases.

\begin{tabular}{lll}
\hline family & type of data & size \\
(filename) & &
\end{tabular}

TART:

tartnd

tartppd

gamdat

cross

*******

******

******

gen2000

EOS:

eop

eos4

pretlib

NDF:

ndflib

ndf $1, \ldots$, ,ndf7

access routines to $n d f 1, \ldots, n d f 7$

neutron interaction cross sections

neutron induced gamma-ray cross sections

gamma-ray interaction cross sections

multi-band neutron interaction cross sections

atomic data projectile independent data

all particle Monte Carlo multigroup (neutron, charged)

all particle energy dependent (gamma, electron)

access libraries (under development)

equation of state data

access routines to eop

energy-density tabular eos data and access routines

545280

524288

741376

12288

2635847

interaction data

TXS:

txslib access routines to txslib00,...,txslib08

txslib00,..,08 tabular freq. dep. LTE opacity data

163840

9437184

other:

dca

(ascii forms exist) atomic physics data

supporting:

pptab

bdfls

cpdf

tdf

evaluated:

endl

egdl

ecpl

epdl (ascii) daughter list

(ascii) basic nuclear data

charged particle Monte Carlo data

thermonuclear data file (under development)
4387

24576

131072 
Sizes in the above table are in 64-bit words.

In the above table, $* * * * * *$ indicates that the file is under development and has not yet been released to public. In addition, gen 2000 is also under development. The library, pretlib, is used only by B Division and its conversion is not yet funded. If funded, this project would also include pretlib. Also, cpdf is used by several production codes and is to be replaced by J. Rathkopf in the near future. Finally, Hopi (written by Richard White) which produces tabular opacity output which is then processed by PacMan (written by Robert Dye) and by Emily (an unfinished utility by William Warren) are used to generate txs libraries. Since these three codes are unsupported and no decision has been made to support them, the future of the opacity system is currently uncertain and can not be assured by this porting project. However, the current txs capability will be ported to the workstations by this project. Codes that generate the eop database, including h0eos, eopall, hmctab, hcon and edgar will not be ported by this project.

Per request, production codes are of immediate concern to this project.

\subsection{Computational Accuracy}

For the workstation environment to be a useful scientist's tool, it must maintain at least the same degree of accuracy the supercomputers have traditionally provided. We will, however, be able to tolerate some loss of exponential range. For the workstations, this means that double precision (64bit) floating point will be required. In addition, since workstations, in general, only support 32-bit integers, provisions will have to be made for all integers to be 32-bits. The corresponding Cray databases and accessing libraries will continue to use Cray single precision (64-bit) floating point and (64-bit) integers. In some circumstances it may be possible to get by with 32-bit floating point on the workstations. However, for simplicity, this project will uniformly deliver 64-bit floating point results.

\subsection{Computational Speed}

To be accepted by the users and code developers alike, production codes using the ported physical databases must perform their functions in a timely manner. The overhead associated with the physical databases must be acceptable. Fortunately, the physical databases are generally accessed only at problem generation and possibly at restart times. Thus, some non optimal performance in the database accessing routines and in the libraries can be tolerated provided that the inefficiencies are paid for only a few times per calculation. Access to a remote physical database over a network will probably prove too slow and will require that at least some large fraction of the database exist locally on each workstation. Performance considerations may well force hand-coding of some of the look-up routines. This will be assessed after the conversion.

\subsection{Cray and Workstation Portability}

The portability issue applies to both the physical database and the accessing library routines.

To reduce maintenance problems resulting from supporting multiple computer architectures, the accessing library routines source code will be converted to a more portable syntax based on Fortran 77 with limited ( $\mathrm{Cft}$, not $\mathrm{Civic}$ ) pointer support. Although adopted in Fortran $9 \mathrm{x}$ with a different syntax, limited pointer support in Fortran is currently available in Sun, Dec, Mips, Sony and IBM workstations. The use of conditional compilation based on architecture will be minimized; the implementation of a library providing virtual or generic operating system functions is preferred. Inherently, non-portable constructs will be isolated into a few support library routines. Where this is not meaningful, owing to the unique requirements of a particular accessing library, all architecture dependent routines will be short and clearly marked.

To insure that users have access to exactly the same physical databases as their Cray using cousins, it is proposed that the physical databases on the workstations be a bit for bit identical copy of the 
corresponding Cray database. It will be an easy matter to ftp any and all required data from the Crays and to verify that they are identical to that on the Cray. More automated procedures might also be used.

Routines from auxiliary libraries (Fortlib, Baselib, etc.) used by the accessing library routines will be ported or implemented on the workstation. Maintenance of a single portable version of a library is desired and will be required if at all possible. Recognizing that the libraries may be used by others, the current Cray interface will be maintained, if at all possible. If not, the Cray interface (external reference specification) will be changed to agree with that of the workstations.

\subsection{Archival Requirements}

Physical databases will be maintained, protected and archived by the current responsible owningdeveloping-generating group, in accordance with current procedures and standards.

Once converted and verified, revised accessing library routines will be returned to the original owningdeveloping-generating group, which will be responsible for future updates and distribution, as well as archiving. In addition, copies will also be given to the Meg Centurion ("Librarian") for inclusion in the Meg system update and archival procedures.

\subsection{Code Developer and Data Manager Education}

Code developers and data managers responsible for the various data will be kept informed of plans and progress in the physical database porting project. Periodic meetings to help set direction and to inform will be scheduled. The following Table 2 contains the names of those known to be responsible for the various physical databases.

TABLE 2. The various physical databases.

\begin{tabular}{lll}
\hline $\begin{array}{l}\text { family } \\
\text { (filename) }\end{array}$ & type of data & responsible \\
& persons
\end{tabular}

TART:

$\begin{array}{lll}\begin{array}{l}\text { tartnd } \\ \text { tartppd } \\ \text { gamdat }\end{array} & \text { neutron interaction cross sections } & \text { Roger White/T. Perkins } \\ & \text { neutron induced gamma-ray cross sections } & \text { Roger White/T. Perkins } \\ \text { gamma-ray interaction cross sections } & \text { Red Cullen/T. Perkins/ } \\ \text { cross } & \text { multi-band neutron interaction cross sections } & \text { E. Plechaty } \\ * * * * * * & \text { atomic data projectile independent data } & \text { T. Perkins/E. Plechaty } \\ * * * * * & \text { all particle Monte Carlo multigroup (neutron, charged) } & \text { T. Perkins } \\ * * * * * * & \text { all particle energy dependent (gamma, electron) } & \text { T. Perkins/J. Rathkopf } \\ \text { gen2000 } & \text { access libraries (under development) } & \text { J. Rathkopf }\end{array}$

EOS:

$\begin{array}{lll}\text { eop } & \text { equation of state data } & \text { D. Young/E. Corey/ } \\
\text { eos4 } & \text { K. Skinnell } \\
\text { pretlib } & \begin{array}{l}\text { access routines to eop } \\
\text { energy-density tabular eos data and access } \\
\text { routines (used primarily by B Division) }\end{array} & \text { L. Chase } \\
& \text { R. Grover/G. Haggin/ } & \text { Kem Hainebach }\end{array}$


NDF:

$\begin{array}{lll}\text { ndflib } & \begin{array}{l}\text { access routines to ndf1,...,ndf7 } \\ \text { ndf } 1, \ldots, \text { ndf7 } 7\end{array} & \begin{array}{l}\text { Roger White } \\ \text { interaction data }\end{array} \\ \text { Roger White }\end{array}$

TXS:

txslib

access routines to txslib00,...,txslib08

(written by R. Litterst)

none assigned

txslib00,..,08 tabular freq. dep. LTE opacity data

none assigned

Hopi (written by Richard White) output

processed by PacMan (R. Dye) and by the

unfinished emily (W. Warren)

supporting:

$\begin{array}{lll}\text { pptab } & \text { (ascii) daughter list } & \text { T. Perkins } \\ \text { bdfls } & \begin{array}{l}\text { (ascii) basic nuclear data } \\ \text { charged particle Monte Carlo data (to be }\end{array} & \begin{array}{l}\text { T. Perkins } \\ \text { unknown }\end{array} \\ \text { tdf } & \begin{array}{l}\text { replaced by J. Rathkopf) } \\ \text { thermonuclear data file (under development) }\end{array} & \text { Roger White }\end{array}$

other:

dcalib (ascii forms exist) atomic physics data Y. Lee/Lasnex

evaluated data (not read directly by most production codes):

endl (ascii forms exist) evaluated neutron $\quad$ Roger White

$\begin{array}{lll}\text { egdl } & \text { (ascii forms exist) evaluated gamma } & \text { R. Cullen/E. } \\ \text { ecpl } & \text { (ascii forms exist) evaluated charged particle } & \text { Roger White }\end{array}$

$\begin{array}{lll}\text { epdl (ascii forms exist) evaluated photon } & \text { R. Cullen/T. Perkins }\end{array}$

The following Table 3 contains the names of our informal review group.

TABLE 3. Members of the informal advisory and review group.

\begin{tabular}{lll}
\hline name & L-code & extension \\
\hline David Young & L-299 & \\
Ellen Corey & L-299 & $2-7231$ \\
Roger White & L-298 & $2-1358$ \\
Dave Resler & L-298 & $3-9668$ \\
Robert Howerton & L-298 & $2-4054$ \\
Lila Chase & L-298 & $2-4086$ \\
David Lappa & L-298 & $2-5125$ \\
Dick White & L-298 & $2-4097$ \\
Roy Swiger & L-298 & $2-4129$ \\
Ted Perkins & L-298 & $2-4061$ \\
Red Cullen & L-298 & $3-7359$
\end{tabular}




$\begin{array}{lll}\text { Jim Rathkopf } & \text { L-298 } & 2-4602 \\ \text { Paul Dubois } & \text { L-472 } & 2-4237 \\ \text { Ron Litterst } & \text { L-321 } & 2-4147 \\ \text { Steve Hornstein } & \text { L-16 } & 2-4698 \\ \text { George Zimmerman } & \text { L-18 } & 2-5429 \\ \text { Dale Nielsen } & \text { L-18 } & 2-4654 \\ \text { Randy Christensen } & \text { L-35 } & 3-3054\end{array}$

\subsection{Programming Standards}

Programming standards are important in insuring maintainable and well documented code. The standards of this project are those of Meg as outlined in the Meg Programming Style and File Manual, UCID-19662, January 13, 1983.

\section{Implementation Order}

The Physical Database Port to Workstations project will deliver major sub-components in phases so that early workstation support is available to candidate users. Candidate users include O-Division, the Lasnex group, the Meg group, and the Nuclear Data group. Others may be added as needed or requested. Delivery includes making available the necessary accessing libraries as well physical databases, necessary documentation and appropriate notification of availability.

Implementation phases will also allow the project approach to be changed if that proves necessary.

The ndf library products will be the first delivered product. The txs library products will be the second delivered product.

The succeeding deliverables will include the other physical database packages as they are ported. The last package to be completed will be the eos family, since this package represents the largest effort.

\section{Alternative Approaches}

Seven approaches have been identified for consideration in the porting effort. It is not likely that all physical databases and their supporting access routines will be ported with the same approach or with the same level of difficulty. Thus, we may be forced to use one approach with one database and a different one for yet another database. The following are the seven approaches:

1. Make the data bases ASCII. (Some data bases are already ASCII and some have ASCII versions. They do not require further conversion effort.)

2. Use a commerciai ciaia base manager which would handle transparently both the file organization and machine dependence.

3. Have a centralized data base manager which would be the distributed version of the above.

4. Convert the binary data bases from Cray binary to IEEE binary.

5. Write the binary data bases in IEEE mode, initially. This would involve converting the codes that generate the data bases to run on the target machines. This was the approach (more or less) followed in moving from the 7600 s to the Crays. 
6. Make the access libraries machine independent and have them read the Cray binary data and convert it to native (IEEE) form before returning to the user.

7. Make the codes that generate the data bases write the data in IEEE format, rather than in Cray format. Unlike 5 above, this would not involve converting the generating codes to workstations. Rather, the Cray versions would be taught to write IEEE format data.

\section{Criteria for Evaluating Approaches}

The following criteria have been suggested for evaluating the approaches used in the various porting efforts:

1. The amount of effort, short term and long term.

2. Verification of data across all machines.

3. Conceptual simplicity.

4. Ainount of machine independence.

5. Impact on production codes.

6. Impact on generating codes.

7. Ability to debug.

This list needs to be expanded and amplified as experience is gained.

\section{Plan of Attack}

\subsection{Executive Overview}

Develop Preliminary List of Physical Databases and their Accessing Library Routines

Develop Preliminary List of Responsible Owning Individuals and Organizations

Develop Preliminary List of "Concerned" Individuals for Overview Panel

Develop Preliminary List of Porting Approaches

Develop Preliminary List of Evaluation Criteria

Develop Preliminary List of Workstation Hardware and Software

Identify Individuals to Work on the Physical Database Porting Project

Order Necessary Workstation Equipments

$3 \mathrm{X}$-window Stations

1 Sun Sparcstation 2

Develop Preliminary Tool Set

create one-time-use Fortran translator or use Precomp 
write routine to convert 64-bit Cray reals to 64-bit IEEE reals write routine to convert 64-bit Cray integers to 32-bit IEEE integers write necessary Fortlib/Baselib routines for the workstations write rdabs for the workstations

Port ndflib to the Workstation

Develop Project Cost and Schedule

Obtain Management Approvals

Review Experience and Approaches with Informal Review Panel

Participate in Quarterly Reviews

Brief Management on Progress

Develop Additional Tools

Review any Required Changes in External Specifications with Users and Owners

Port remaining Physical Databases and Accessing Routines to the Workstation

Return Ported Routines to owning Groups

Prepare Documentation Especially, any Changes in External Specifications Indicate, any necessary changes

Otherwise, go with the Documentation Supplied by the Owning Group

Disseminate Information to Using Groups prepare releases when any useful subset of databases is available

\subsection{Port Intrinsics}

A port includes the following activities for each physical database:

make whatever changes are needed, if any are necessary

A port includes the following activities for each physical database access library:

obtain source for conversion

apply one-time-use translator or Precomp, if needed

hand-edit non-portable constructs

test portability modifications on Cray run tests using unaltered Cray code, save results run tests on modified Cray code, save results compare results of unaltered and modified versions

build workstation version compile and load 
implement missing auxiliary library routines

test portability on workstations

run test on workstation code, save results

compare results of modified Cray and workstation versions

document relevant details for inclusion in various places

\subsection{Milestones}

A schedule of milestones is provided assuming that 2 FTE commence work October 1, 1990, that they do not have other miscellaneous duties, and that support will be available for at least a year. At the present time, we expect about .8 FTE from Lila Chase, 1 FTE from Clifford Rhoades and the remaining .2 FTE from Ron Litterst. The estimated schedule will be reviewed in March after members of the team have gained some additional hands-on experience. The schedule will be revised at that time.

To help quantify time estimates required for repetitive operations the following information was used:

- removing Precomp dependency and converting Cray source to more "standard" syntax took 2 weeks for the 2500 line ndflib physical database accessing package.

- an additional 3 weeks were required to find and correct all the semirecursive calls permitted by Civic but not supported by $\mathrm{Cft} 77$. (Semirecursive calls are those in which a routine directly or indirectly calls one of its own entry points.) Debugging was done using the standard Meg code.

- check-out on the Sun Sparcstation 2 has been delayed owing to delayed delivery of the hardware

The major Milestones are:

Scheduled Actual Event

10/01/90 09/04/90 Hold Advisory Panel Meeting

- identify all required physical databases and access routines

- review porting schemes

- develop evaluation criteria

10/01/90 09/05/90 Place Order for Sun Sparcstation 2 and X-windows Terminals

10/01/90 11/28/90 Obtain Agreement on Project Funding

10/15/90 09/19/90 Document Advisory Panel Meeting

- confirm voluntary assignments

- confirm meeting conclusions

- disseminate to check for error

11/15/90 11/15/90 Complete Gaining Experience Porting ndflib

12/03/90 12/11/90 Write First Draft of Project Plan

$12 / 13 / 90 \quad 12 / 17 / 90 \quad$ Receive Sun Sparcstation 2 and X-windows Terminals 
01/07/91 12/09/91 Sun Sparcstation 2 and X-windows Terminals Available for Classified

03/01/91 04/03/91 Obtain X-windows Terminals for Ron Litterst and Richard White

03/07/91 04/09/91 X-windows Terminais Available for Classified

03/07/91 07/09/91 311 Office for Ron Litterst

--/--/- Mostly Ported Lasnex Generator Available

--1--1- Mostly Ported Meg Generator Available 


\section{Appendix: Currently Known Constraints}

1) Cray library access, except for EOP and EOS4, are not subject to modification. That is, the external reference specification will have to remain as it currently is.

2) SPARC architecture machines (i.e. Sun) are the only classified UNIX systems available to this project. Therefore, it will not be possible to verify machine independence without making at least one other architecture (such as MIPS, DEC or IBM) available. Estimated cost is under $\$ 25 \mathrm{~K}$.

3) Requirements for graphical output, such as postscript, displaj postscript, FR80 etc., need to be established elsewhere so that the designers can have an adequate visualization system. 
Appendix: Miscellaneous Details which impact time estimates

1) Ndflib has 2438 source lines.

2) Txslib has 2940 source lines, not including 309 lines in the caching routine and a cal (assembly) routine of 104 lines.

3) The number of source lines for the remaining libraries will be indicated when determined. 


\section{Appendix: Eos4 Details}

EOS4 is the access library to the EOP equation of state tables. The goal of this project is to provide an access library to the EOP equation of state database. The code conversion and documentation will be done by Lila Chase.

\section{Characteristics of Current EOS4 on N/LTSS}

- Both the old (and no longer maintained) EOS and the maintained EOP databases are supported.

- User interface is via a large common block; user is instructed to set various variables in that common block. Options are chosen by setting the appropriate bit in various flags.

- User calls each routine that needs to allocate space twice, once to calculate the space, and again after the space has been allocated.

- The access library consists of general and special selectors, forward scalar and vector lookups, and inverse scalar and vector lookup.

- The interface requires the user to set vector descriptors to all the required arrays. A descriptor is a 64 bit structured Cray word consisting of a 32 bit length and a 32 bit bit address. Thus, the largest word address that will fit in 32 bits is $2^{* * 32 / 64}$ or $67,108,864$. The Y machine (YMP) currently has $32 \mathrm{M}$ words of memory, where $\mathrm{M}=1,048,576$. Future memory upgrades, such as a proposed $128 \mathrm{M}$ word upgrade, would cause the notion of descriptors to become obsolete.

- The same variables are often used for dual purposes, depending on whether a certain bit of a flag is set. The variable could be regarded as a scalar or as a descriptor to a vector. Because descriptors have to be replaced by pointers and lengths, such a dual functionality is no longer possible. Also, the data types are different.

- To conserve space, some options use halfwords.

- Also to conserve space, a fixed length (1024) internal FORTLIB common block, ZVCACHES, is used for temporary storage. This use imposes an upper limit of 64 on the number of EOS numbers.

- The current implementation makes extensive use of LRLTRAN extensions and other sitedependent constructs:

-- In-line assembly code

-- Vector syntax, such as $\mathrm{X}=\mathrm{Y}(\mathrm{OFFSET}) * \mathrm{Z}$ QVSCAT

-- STACKLIB routines: Q8SUM, Q8CMPRS, Q8RBM, Q8WBM, QZTOIL, QVGATH,

-- BIT and STRUCTURE data types

-- Jim Kohn's NOGOTOS macros

-- Macros with arguments; LASLIB macros

-- Alphanumeric labels 
-- Non-standard DATA statements: DATA $(X=\ldots)$

-- Use of .INT., .UN., .SHR., .SHL., .REAL., .INTEGER., .DES.

-- Use of CIVIC OP TION statement to toggle certain options

-- Non-portable methods for storing and fetching data

- Use of ABSOLUTE for direct access to an address

- Use of offsets from some array in common, where some inline function is used to compute the offset.

- There are remnants of STAR coding that should be removed. For example, certain data is stored on even word boundaries.

- Coding is often cryptic with no explanation of intent. For example, INDEX = INDX .SHL. 3 was really intended as INDEX $=$ INDX *8.

- There are many options to set multipliers, limits, and so on.

\section{Approach}

- Keep as much of the original functionality as possible, but rewrite the interface. Changes are necessary anyway, because of the limitations of descriptors.

- Because a change is necessary anyway to the user interface, take the opportunity to improve the code by

-- Removing one big common block approach and let the the user only have access to that which he needs. Subroutines would be called with argument lists.

-- Let EOS4 allocate and deallocate space it needs, rather than have the user allocate it. Several two-call routines would then be reduced to one-call. The user can replace the library EOSALLOC and EOSDALLOC with their own, if they wish to their own scheme for memory management.

-- Provide a CHARACTER error message EOS4ERR in addition to the current integer error flag IERRFLAG. Change all user-callable subroutines to functions which return IERRFLAG for its value.

-- Clean up communication between the modules.

-- Remove STAR-dependent coding.

-- Remove half' , ord options.

-- Remove handling of EOS data base.

-- Replace ZVCACHES with an allocatable EOSSPC.

-- Use IMPLICIT NONE and whatever else is needed to create safe, maintainable code. 
- Make the code portable.

-- Remove dependence on PRECOMP. Use INCLUDE files instead of cliches. Eliminate cliches with arguments.

-- Minimize shifting and masking operations.

-- Replace all vectors with pointered arrays, and replace all vector operations with doloops. Although array syntax is defined in Fortran 90, it will be awhile before workstation compilers support it.

-- Replace the hand-coded lookup with Fortran code. Look at the efficiency issue again when SCF is converted to UNICOS. The forward lookup may again need to be hand-coded.

- Where possible, make the code re-entrant for multiprocessing.

- The selector collects statistics on the EOP numbers used and the ids (user numbers) of the users. H-Division requires this information for updating the tables. A replacement mechanism is provided for statistics collection.

\section{Timetable}

Scheduled Actual

12/28//90 12/01/90 Selector: Conversion of General Selector to CFT77 with minimal use of

for statistics BASELIB (for I/O to absolute file). Replacement mechanism selector. collection. Conversion of that portion of the tester that calls

01/30/91 01/15/91 Workstation Development: Move code development from Cray to absolute $\quad$ I/O with portable version.

02/27/91 03/28/91 User Interface: Propose a new user interface, and iterate with users until acceptable.

03/30/91 03/15/91 EOP on Workstation: Change the general selector to read and convert the Cray data as required.

04/30/91 04/19/91 Forward Lookup: Conversion of Forward Lookup. Conversion of that portion of the tester that calls the forward lookup.

05/30/91 06/30/91 Inverse Lookup: Conversion of Inverse Lookup. Conversion of that portion of the tester that calls the inverse lookup.

06/30/91 07/15/91 Additional (Optional Routines): Upon user request, conversion of special selectors (preamble and header selectors).

$07 / 30 / 91$

User Documentation: Update user documentation.

$08 / 30 / 91$

User Testing and Debugging.

$09 / 30 / 91$

Installation and Checkout. 


\section{Deliverables}

- Source and binary for EOS4 access library to EOP data base.

- EOP data base for workstation (if that approach is taken).

- User documentation. 


\section{Appendix: TXS Details}

\section{Introduction}

TXS in general refers to a fairly large software collection of programs, libraries and databases which support the opacity effort at LLNL. The TXS software system begins with codes such as HOPE, XSN and HANK which calculate the raw frequency-dependent opacity data as functions of temperature and density. The raw output of these codes plus any experimental data are processed by HOPI. HOPI, written by Richard White, is a tool to allow review, plotting and thinning of the opacity data. It can be used by users who are interested in some particular opacity or by the opacity database creator. Multiple outputs of HOPI are organized and written to the public files (txslib00, txslib01,..., txslib08) by CLYDE, writter by Bob Dye. To enable conversion to a more flexible public file format and to allow conversion to NLTSS, Bill Warren has written EMILY, as a replacement for CLYDE. EMILY was not completed before Bill was promoted to work in another program at the Laboratory. The goal of the TXS project is to provide an access library (the TXS software system, also called TXSLIB) which can read the new TXS public library files and which can also run on the workstation. This is a more difficult task than that concerning NDFLIB, because TXSLIB writes a problem library file which only contains the data needed by gererated problem. The code conversion and documentation will be done by Ron Litterst.

\section{Background}

TXS (Tabular Cross Sections) is a system used to tabulate and interpolate frequency dependent photon absorption cross sections. TXS supplements or replaces "in-line" analytic cross section calculations such as XSN [Lokke(1975), Zimmerman and More (1980)]. TXS is much faster than XSN. It permits use of databases derived from atomic models more sophisticated than XSN's.

The TXS cross section library tabulates cross section as a function of photon energy, temperature, and ion molar density. In the standard TXS cross section library, there is a single table for each element. Cross sections for mixtures are calculated from the elemental cross sections. (The system does not, however, preclude constructing and using independent tables for mixtures.)

The energy range cov red by the tables is divided into bins bounded by energies. The tabulated cross section is an average value of the frequency dependent cross section in the group interval. The spectral resolution of TXS's (public) Library (a collection of files to conform to public space limits) is less than can be achieved with XSN. However, the resolution is greater than that given by the energy group structures used in most transport calculations. User's who require higher resolution can construct private TXS libraries.

TXS is compatible with codes which use XSN. Calling sequencies are so like XSN's that conversion of user codes from XSN to TXS is straightforward.

The TXS cross section library occupies about ten million (64-bit) words. However, the user's RAM (random access memory) dedicated to tables may be as small as a few thousand words. This reduction is achieved as follows. First select, from the TXS library, only those elements needed in the user's calculation. Then, average the library cross sections and map them into user specified energy bins (groups). (Usually, these groups have less resolution than the TXS library.) Then, record these reduced tables on disk in the same format as a library file. This TXS Problem File is usually much smaller than the TXS (public or private) Library File, from which it is derived. It is written in the same format as a TXS library and rence is accessible for analysis by TXS utility codes. Finally, transfer, from the TXS Problem File to RAM, just those parts of tables which are needed, when they 
are first needed.

These parts of tables are kept in a memory "cache" whose size is controlled by the user. Tables read into the cache are retained there until the cache is filled. Then, they are displaced by currently needed parts of the tables. The more memory the user dedicates to the cache, the less often tables will be read from disk and the more efficient becomes the table look-up scheme.

TXS includes software for performing all the above operations.

In addition, the program HOPI (Histogramic OPactity Integrator) permits any user to do any of the followin operations.

Plot and display cross section data from various source codes.

Use data from various sources to make tables for the TXS system.

Plot and display the cross sections tabulated in any TXS library.

View the results of interpolation in the TXS tables.

View the results of mixing elemental tables.

Edit tables and combine tables based on various sources.

Display mean cross sections for arbitrary group structures.

Display mean cross sections for several types of means.

Overlay and compare any of the plots described above.

Make hardcopy or viewgraphs of any plots described above.

TXS uses the code CLYDE which reads tables made by HOPI and adds them to any designated TXS library. This permits users to assemble private libraries of tables tailored to their own needs in the event that the public TXS library files is inadequate for these special needs. The following figure shows the existing TXS system. The right hand lower portion of the diagram, labeled "Possible Future Work for a New System," shows a planned system (described above) whose implementation is outside the scope of this project. The arrows on the lines show data flow with the point showing the direction in which the data flows. 
Existing TXS Software System

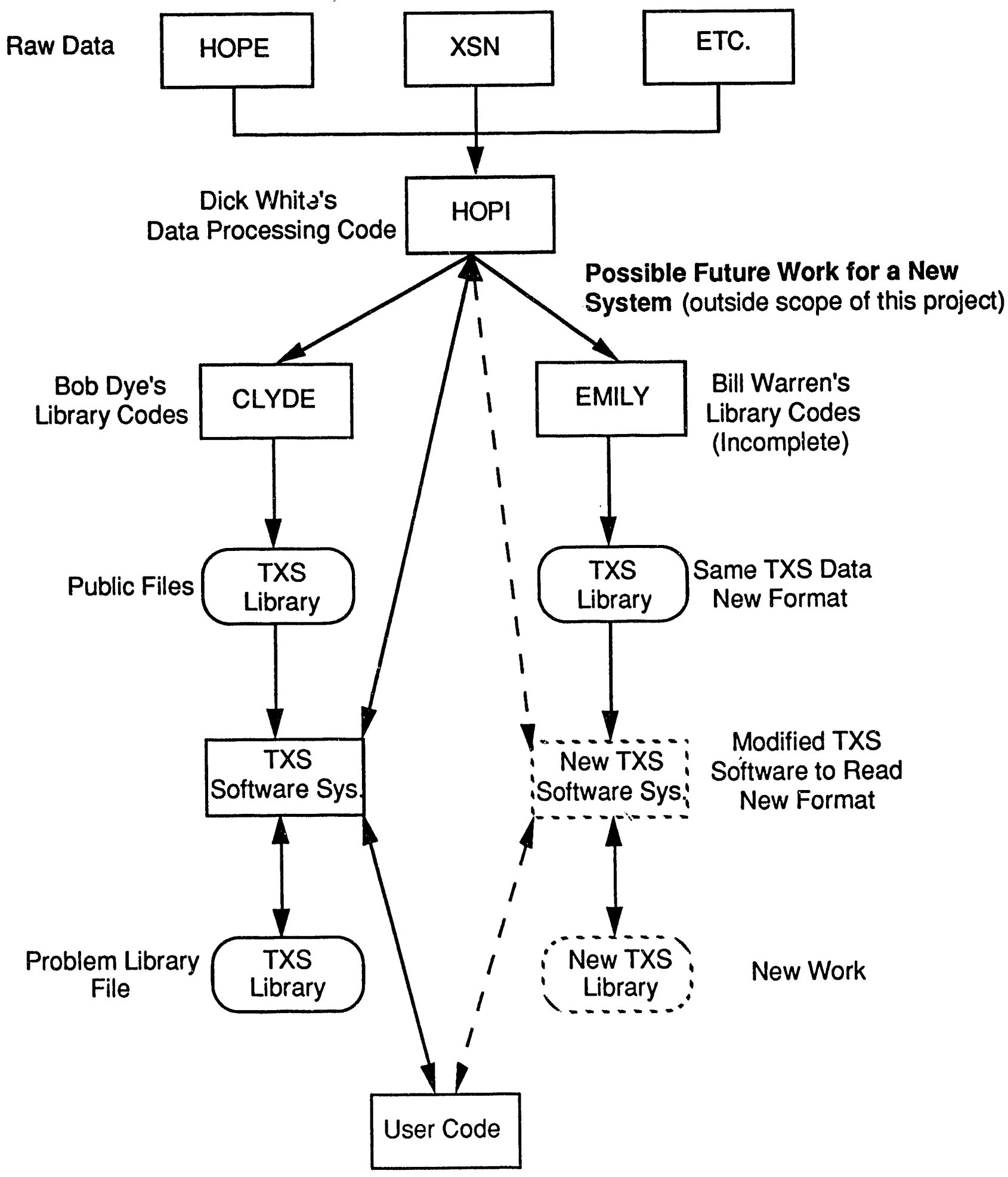




\section{Characteristics of Current TXS System on N/LTSS}

TXS is a self $r$ ontained software system that resides in the publis file system on all Critys, in the form of an independent binary (object) build library as the first file in a LIB file named TXSLIB.

To use the TXS System a user code must first write three subroutines that interface between the user code and the TXS software. These routines pass information and request opacity data. The user code merely reloads naming TXSLIB as an independent build library, thus, loading the required TXS software.

The TXS Public Library contains the elements needed by the Laboratory major production codes and the majority of the data is packed in half words to save disk space.

TXS is faster and more accurate than the XSN method it replaces. The TXS software system was developed to be portable from one production code to another. Therefore, subroutine agquments are used instead of common to pass all information between user code and TXS. Also, all dynamic storage required by TXS is supplied by the user code, and TXS writes no error messages, but reports errors through argument lists to the user code.

All source and binary files are contained in the public Lib file named TXSLIB, which contains the the TXS independent binary (object) build library, as the first file, and a controllee named LIBCK to check the public TXS library family of files for errors.

Backups to all TXS software and library files can be found in mass storage in the take directory, $.535200:$ txsn.

The TXS software takes advantage of LRLTRAN extensions, but not to a large extent. The used extensions are mostly in the areas of cliche macros, word packing and $\mathrm{V} / \mathrm{O}$. The software is compiled under CIVIC and loaded using BUILD and LDR.

\section{Approach for Converting to UNIX Systems}

- Make changes in the current system necessary to keep the source code the same for both N/LTSS and UNIX without changing functionality.

- Make required changes to the source code so it can be compiled by CFT. This includes replacing CLICHES with INCLUDE statements, removing MACROS, replacing nonstandard LRLTRAN constructs with equivalent CFT constructs while retaining functionality, review all SHIFT and MASK operations, clean up INTEGER and CHARACTER usage, etc.

- At the same time clean up communications between TXS routines, add prologues and inline documentation to all routines, and update and refine the TXS users external document.

- Get all TXS source code to compile under CFT and produce a new CFT independent binary (object) build library.

- Use the MEG code as a test vehicle to make sure that the new TXS software system, compiled under CFT, still produces the same, correct answers.

- After this phase is complete, move to the UNIX machine and transfer the Cray TXS library files so that they reside on the UNIX machine as Cray absolute files.

- Next rewrite the TXS I/O routines so that they read and select elements from the Cray type 
files, and write a UNIX type run time problem library file. Special attention will be paid to insure that the source files are compatible between N/LTSS and UNIX.

- Design and write a test code that will exercise the UNIX TXS system, in a way similar to the MEG usage on the Crays. This will help show the correctness of the revised TXS software and TXS library.

- When this is accomplished, then move the new TXS sources back to the Cray, compile with CFT, load it into MEG and run the test problems to show that no new errors have been introduced.

\section{Timetable}

Scheduled Actual

07/26/91 Come up to speed on the new platform, operating system and software development tools.

08/09/91

Make required changes to TXS source code to allow compilation by CFT.

$08 / 23 / 91$

Clean up communication between TXS routines, add prologues and inline documentation.

$08 / 30 / 91$

Use MEG to check out the revised TXS routines.

$09 / 13 / 91$

library.

Rewrite the TXS I/O routines so that they read and select elements form the Cray type absolute files and write a UNIX type run time problem

$09 / 26 / 91$

Complete test code and run tests under UNIX.

$09 / 30 / 91$

Run updated software on the Cray to insure that no new bugs were introduced.

\section{Deliverables}

- A TXS system that runs under both UNIX and N/LTSSS.

- TXS source code that is cleaned up and which contains adequate internal documentation.

- TXS public database for workstation.

- User documentation. 
Appendix: External Referen乞 Specitication of Supporting Library

Descriptions of following supporting routines is provided for reference: ieee, eeei, eeeil, bcast, move, freeus, Inblnk, rdabs, rdabspn, iowait, iostatus, empty, second, skip, setbuf, getspace, relspace, shiftr, shiftl, shift, zmovebit, zmvbit, compl, or, and, luf, and minaf. The routines, lnblnk, iostatus, empty, second, getspace, relspace, shiftr, shiftl, shift, zmovebit, zmvbit, compl, or, and, luf, and minaf are new to the March 28, 1991 release of the supporting library (portlib). The routines, gather, scopy, sdot, ssum, and dpint are new to the July 29, 1991 release of the supporting library. All of these routines are currently available. 

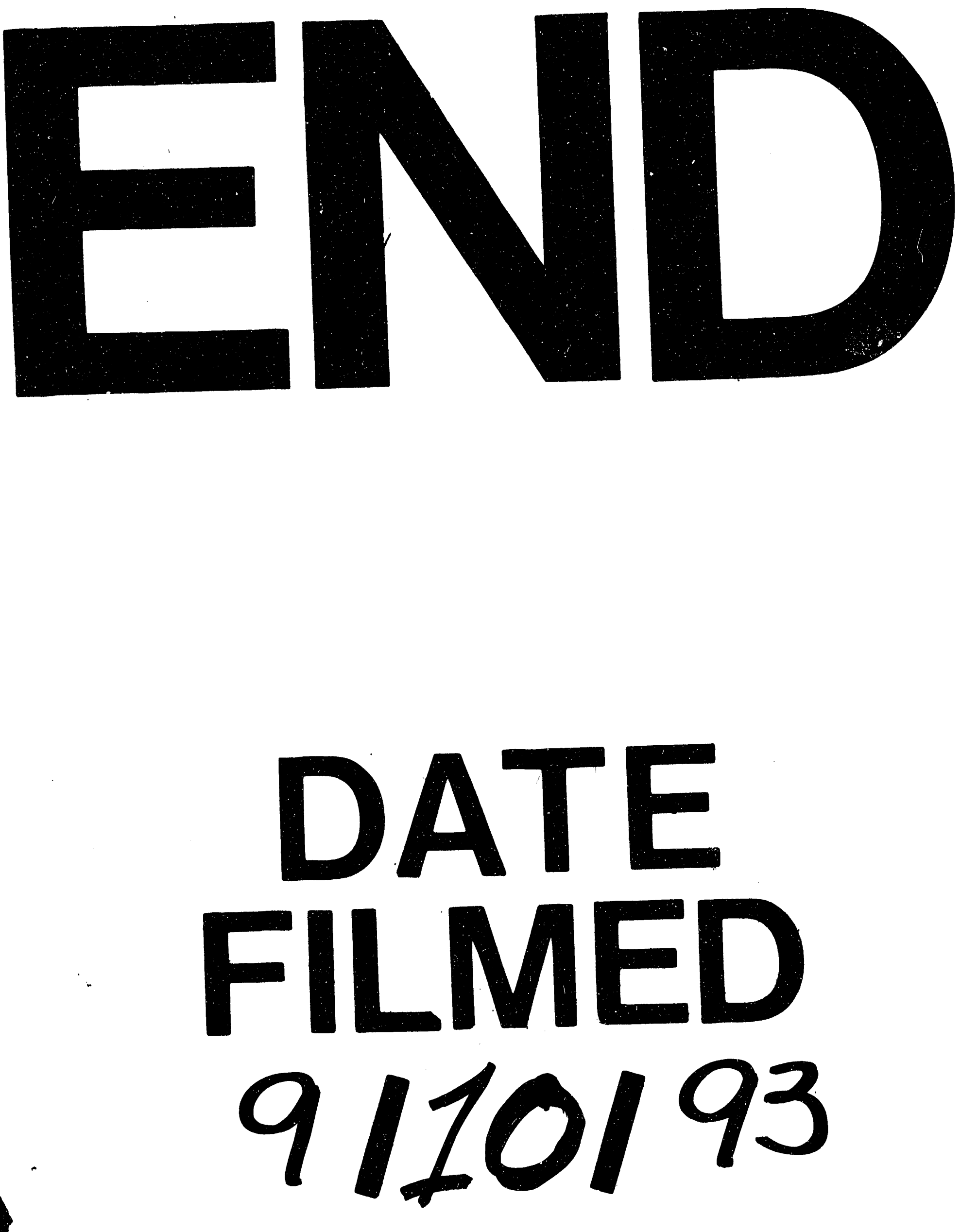